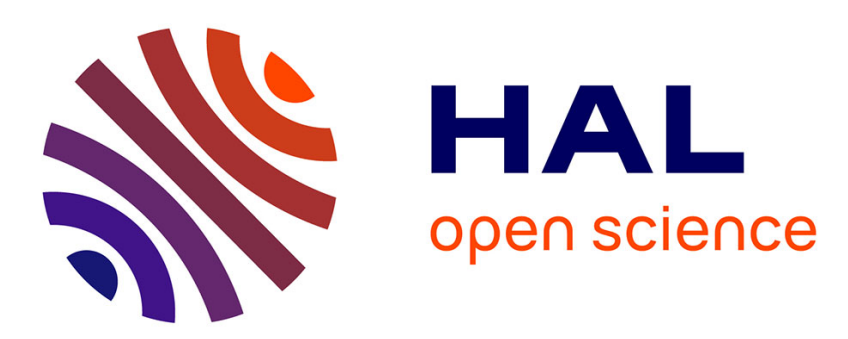

\title{
Formulation and approximation of quasistatic frictional contact
}

\author{
Marius Cocou, Michel Raous, Elaine Pratt
}

\section{To cite this version:}

Marius Cocou, Michel Raous, Elaine Pratt. Formulation and approximation of quasistatic frictional contact. International Journal of Engineering Science, 1996, 34 (7), pp.783-798. 10.1016/00207225(95)00121-2 . hal-03178360

\section{HAL Id: hal-03178360 \\ https://hal.science/hal-03178360}

Submitted on 14 Apr 2021

HAL is a multi-disciplinary open access archive for the deposit and dissemination of scientific research documents, whether they are published or not. The documents may come from teaching and research institutions in France or abroad, or from public or private research centers.
L'archive ouverte pluridisciplinaire HAL, est destinée au dépôt et à la diffusion de documents scientifiques de niveau recherche, publiés ou non, émanant des établissements d'enseignement et de recherche français ou étrangers, des laboratoires publics ou privés. 


\title{
FORMULATION AND APPROXIMATION OF QUASISTATIC FRICTIONAL CONTACT
}

\author{
MARIUS COCU and ELAINE PRATT \\ Laboratoire de Mécanique et d'Acoustique-C.N.R.S. 31, chemin Joseph Aiguier, \\ 13402 Marseille cedex 20, France and Université de Provence, Marseille, France \\ MICHEL RAOUS \\ Laboratoire de Mécanique et d'Acoustique-C.N.R.S. 31, chemin Joseph Aiguier, \\ 13402 Marseille cedex 20, France
}

\begin{abstract}
A quasistatic unilateral contact problem with a non-local friction law is considered. We propose a new variational formulation of this problem consisting of two inequalities. By applying an implicit time discretization scheme, we obtain an incremental formulation which, if the friction coefficient is sufficiently small, has a unique solution for which appropriate estimations are obtained. This incremental solution enables us to construct a solution to the quasistatic problem by establishing the weak convergence of a subsequence of mappings interpolating the incremental solution. An algorithm is derived and a simple numerical example is presented.
\end{abstract}

\section{INTRODUCTION}

We consider a quasistatic unilateral contact problem with a non-local Coulomb friction law $[1,2]$. For such evolutive problems the main difficulty that arises in establishing the variational formulation of the quasistatic problem is the simultaneous presence of the displacement field, which appears in the unilateral contact condition, and of the velocity field which appears in the friction law. Most of the theoretical results on friction concern static contact problems.

For quasistatic problems, results have been obtained by using a regularization of the unilateral and friction conditions. Friction conditions based on the normal compliance model (see $[3,4]$ ) have been studied by Klarbring et al. $[5,6]$ for incremental formulations of quasistatic problems and by Martins and Oden [7] for dynamic problems. The existence of a solution for quasistatic contact problems with a normal compliance law was proved by Andersson [8], using an implicit scheme, and in the presence of a time regularization, by Klarbring et al. [9], by a different technique.

Here both unilateral and friction conditions are strictly imposed and we do not use either penalization or normal compliance. We present in Section 2 a new variational formulation that combines two inequalities: an inequality which represents the unilateral contact condition and an inequality which describes the friction condition under a differential form with the velocity field as test function. The functional background and notations are also given in this section.

A time discretization is adopted in Section 3. We prove that if the friction coefficient is sufficiently small then the equivalent incremental formulation we have derived from this discretization has a unique solution. Appropriate estimations for this solution are then obtained.

Section 4 contains the proof of the existence of a quasistatic solution. Using the incremental solution obtained in Section 3 we construct a sequence of piecewise constant mappings defined on the time interval considered. A set of technical lemmas enables us to establish in the first place that some subsequence of the above sequence has a weak limit and then to conclude by showing that this weak limit is a quasistatic solution. 
Finally in Section 5 an algorithm for computing the incremental solutions is presented and a simple numerical example is given.

\section{QUASISTATIC FORMULATION}

\subsection{The quasistatic unilateral problem with friction}

We consider an evolutive Signorini problem with non-local Coulomb friction. We deal with an elastic body occupying a domain $\Omega$ of $\mathbf{R}^{d}, d=2,3$, with a sufficiently regular boundary $\Gamma=\bar{\Gamma}_{1} \cup \bar{\Gamma}_{2} \cup \bar{\Gamma}_{3}$. The displacements are prescribed on $\Gamma_{1}$ and mes $\left(\Gamma_{1}\right)>0$. An evolutive force density $\varphi_{2}$ is applied on $\Gamma_{2}$. The solid is initially in contact with a rigid fixed support on $\Gamma_{3}$, the evolution of the deformations on this part of the boundary is restricted by a unilateral condition of non-penetration into the support and submitted to friction forces when contact occurs. The possibility of an initial gap $\alpha$ will be introduced in the computations; from a theoretical point of view this introduces no further difficulty as long as $\alpha$ is supposed sufficiently small to be of the same order as the deformation $\mathbf{u}$ as the present formulation is set under a small deformation formulation. On $\Omega$ a volume force density $\varphi_{1}$ is applied.

The given forces $\varphi_{1}$ and $\varphi_{2}$ are sufficiently smooth in space and time, i.e. $\varphi_{1} \in$ $W^{1,2}\left(0, T ;\left(L^{2}(\Omega)\right)^{d}\right)$ and $\varphi_{2} \in W^{1,2}\left(0, T ;\left(L^{2}\left(\Gamma_{2}\right)\right)^{d}\right)$, and we suppose that we may neglect the inertia effect involving the acceleration terms.

We denote by $\boldsymbol{\sigma}=\left(\sigma_{i j}\right)$ the stress tensor, $\mathbf{e}=\left(e_{i j}\right)$ the strain tensor, $\mathbf{u}=\left(u_{i}\right)$ the displacement field, $\tilde{\boldsymbol{\sigma}}$ the contact density forces on $\Gamma_{3}$, and $\mathbf{E}$ the elasticity tensor of the material with the usual properties of ellipticity and symmetry. On $\Gamma_{3}$ we use the following notations for the normal and tangential components of the displacement vector and stress vector:

$$
\begin{array}{cc}
\tilde{\boldsymbol{\sigma}}=\sigma_{\mathrm{N}} \mathbf{n}+\boldsymbol{\sigma}_{\mathrm{T}}, & \mathbf{u}=u_{\mathrm{N}} \mathbf{n}+\mathbf{u}_{\mathrm{T}} \\
u_{\mathrm{N}}=u_{i} n_{i}, & \mathbf{u}_{\mathrm{T}}=\mathbf{u}-u_{\mathrm{N}} \mathbf{n} \\
\sigma_{\mathrm{N}}=\sigma_{i j} n_{i} n_{j}, & \boldsymbol{\sigma}_{\mathrm{T}}=\boldsymbol{\sigma} \cdot \mathbf{n}-\sigma_{\mathrm{N}} \mathbf{n},
\end{array}
$$

where $\mathbf{n}=\left(n_{i}\right)$ is the outward normal unit vector to $\Gamma$.

The problem can be set now by considering the equilibrium equations where $\tilde{\boldsymbol{\sigma}}$ is the unknown contact force, the constitutive equation (elasticity), the kinematic relation (under the small deformations hypothesis), the unilateral conditions and the Coulomb friction law.

Problem $P_{1}$.

Find $\mathbf{u}$ such that for all $t \in[0, T]$

$$
\begin{gathered}
\operatorname{div} \boldsymbol{\sigma}=-\boldsymbol{\varphi}_{1} \text { in } \Omega \\
\boldsymbol{\sigma}=\mathbf{E e} \text { in } \Omega \\
\mathbf{e}=\operatorname{grad}_{\mathbf{s}} \mathbf{u} \text { in } \Omega
\end{gathered}
$$

with the initial condition

$$
\mathbf{u}(0)=\mathbf{u}_{0} \text { in } \Omega
$$

and with the following boundary conditions for all $t \in[0, T]$

$$
\begin{aligned}
& \mathbf{u}=\mathbf{0} \text { on } \Gamma_{1} \\
& \boldsymbol{\sigma} \cdot \mathbf{n}=\boldsymbol{\varphi}_{2} \text { on } \Gamma_{2} \\
& \boldsymbol{\sigma} \cdot \mathbf{n}=\tilde{\boldsymbol{\sigma}} \text { on } \Gamma_{3} \\
& u_{\mathrm{N}} \leq 0, \quad \sigma_{\mathrm{N}} \leq 0, \quad u_{\mathrm{N}} \sigma_{\mathrm{N}}=0 \text { on } \Gamma_{3} \\
& \left|\boldsymbol{\sigma}_{\mathrm{T}}\right| \leq \mu\left|R \sigma_{\mathrm{N}}\right| \text { and }\left\{\begin{array}{l}
\text { if }\left|\boldsymbol{\sigma}_{\mathrm{T}}\right|<\mu\left|R \sigma_{\mathrm{N}}\right| \Rightarrow \dot{\mathbf{u}}_{\mathrm{T}}=\mathbf{0} \text { on } \Gamma_{3} \\
\text { if }\left|\boldsymbol{\sigma}_{\mathrm{T}}\right|=\mu\left|R \sigma_{\mathrm{N}}\right| \Rightarrow \exists \lambda \geq 0 \dot{\mathbf{u}}_{\mathrm{T}}=-\lambda \boldsymbol{\sigma}_{\mathrm{T}} \text { on } \Gamma_{3}
\end{array}\right.
\end{aligned}
$$


where $\mu$ is the friction coefficient and $R \sigma_{\mathrm{N}}$ is a regularization of the normal contact force [10-12].

The notation $|\cdot|$ denotes the absolute value when applied to a scalar and the euclidian norm when applied to an element of $\mathbf{R}^{d}$.

\subsection{Variational formulation}

We shall adopt the following notations:

$$
\begin{aligned}
V & =\left\{\mathbf{v} \in\left(H^{1}(\Omega)\right)^{d} ; \mathbf{v}=\mathbf{0} \text { almost everywhere on } \Gamma_{1}\right\}, \\
W & =W^{1,2}(0, T ; V), \\
K & =\left\{\mathbf{v} \in V ; v_{\mathrm{N}} \leq 0 \text { almost everywhere on } \Gamma_{3}\right\},
\end{aligned}
$$

$\|\cdot\|$ shall denote the norm on $V$ and $(\cdot, \cdot)$ the scalar product on $\left(H^{1}(\Omega)\right)^{d}$,

$\|\cdot\|_{W}$ shall denote the norm on $W$ and $((\cdot, \cdot))$ the scalar product on $L^{2}(0, T ; V)$ (see, e.g. [13]),

$\langle\cdot, \cdot\rangle$ shall denote the duality pairing on $H^{1 / 2}\left(\Gamma_{3}\right) \times H^{-1 / 2}\left(\Gamma_{3}\right)$,

where

$$
H^{1 / 2}\left(\Gamma_{3}\right)=\left\{\left.w\right|_{\Gamma_{3}} ; w \in H^{1 / 2}(\Gamma), w=0 \text { on } \Gamma_{1}\right\} .
$$

Throughout this paper we consider only the Lebesgue measure. We shall therefore omit the integration variable whenever there is no ambiguity (for example we shall set $\int_{\Omega} f$ for $\left.\int_{\Omega} f(x) \mathrm{d} x\right)$.

Before giving a variational formulation of problem $P_{1}$ let us state in which sense the duality pairing $\langle\cdot, \cdot\rangle$ is taken.

It is well known (see e.g. [4]) that if $\boldsymbol{\sigma} \in\left(L^{2}(\Omega)\right)^{d^{2}}$ and $\operatorname{div} \boldsymbol{\sigma} \in\left(L^{2}(\Omega)\right)^{d}$ in the sense of distributions then $\boldsymbol{\sigma} \cdot \mathbf{n}$ belongs to $\left[\left(H^{1 / 2}(\Gamma)^{d}\right]^{\prime}\right.$ and the following generalized Green's formula holds:

$$
\text { for all } \boldsymbol{\psi} \in\left(H^{1 / 2}(\Gamma)\right)^{d},\langle\langle\boldsymbol{\sigma} \cdot \mathbf{n}, \boldsymbol{\psi}\rangle\rangle=\int_{\Omega} \boldsymbol{\sigma} \cdot \boldsymbol{\varepsilon}(\overline{\boldsymbol{\psi}})+\int_{\Omega}(\operatorname{div} \boldsymbol{\sigma}) \overline{\boldsymbol{\psi}} \text {, }
$$

where $\bar{\psi} \in\left[H^{1}(\Omega)\right]^{d}$ and $\bar{\psi}=\boldsymbol{\psi}$ a.e. on $\Gamma$ and $\langle\langle\cdot, \cdot\rangle\rangle$ denotes the duality pairing on

$$
\left[\left(H^{1 / 2}(\Gamma)\right)^{d}\right]^{\prime} \times\left(H^{1 / 2}(\Gamma)^{d} .\right.
$$

Now if $\boldsymbol{\sigma} \cdot \mathbf{n}$ belongs to $\left(L^{2}\left(\Gamma_{2}\right)\right)^{d}$ in the sense of distributions, i.e. $\exists \mathbf{g} \in\left(L^{2}\left(\Gamma_{2}\right)\right)^{d}$ such that

$$
\langle\langle\boldsymbol{\sigma} \cdot \mathbf{n}, \boldsymbol{\varphi}\rangle\rangle=\int_{\Gamma_{2}} \mathbf{g} \boldsymbol{\varphi}, \forall \varphi \in\left(C_{0}^{\infty}\left(\Gamma_{2}\right)\right)^{d},
$$

we define $\sigma_{\mathrm{N}}$ as follows:

$$
\text { for all } w \text { in } H^{12}\left(\Gamma_{3}\right),\left\langle\sigma_{\mathrm{N}}, w\right\rangle=\langle\langle\boldsymbol{\sigma} \cdot \mathbf{n}, \overline{\mathbf{w}}\rangle\rangle-\int_{\Gamma_{2}} \mathbf{g} \hat{\mathbf{w}},
$$

where

$$
\overline{\mathbf{w}} \in\left(H^{1 / 2}(\Gamma)\right)^{d} \text { and } \overline{\mathbf{w}}=0 \text { on } \Gamma_{1}, \overline{\mathbf{w}}_{\mathrm{T}}=0 \text { on } \Gamma_{3}, \bar{w}_{\mathrm{N}}=w \text { on } \Gamma_{3} \text {. }
$$

It is easy to verify that this definition does not depend on the choice of $\bar{w}$ having the above properties.

Now we have the following weak formulation of equation (2.1) using a test function $\mathbf{v}$ 
(homogeneous to a velocity) in $V$. Supposing $\mathbf{u}$ is sufficiently regular with regard to the space variable, Green's formula holds, so that for all $t \in[0, T]$ and for all $\mathbf{v} \in V$

$$
a(\mathbf{u}(t), \mathbf{v}-\dot{\mathbf{u}}(t))-\int_{\Gamma} \boldsymbol{\sigma} \cdot \mathbf{n}(\mathbf{v}-\dot{\mathbf{u}}(t))-\int_{\Omega} \varphi_{1}(\mathbf{v}-\dot{\mathbf{u}}(t))=0
$$

with

$$
a(\mathbf{u}, \mathbf{v})=\int_{\Omega} \sigma_{i j}(\mathbf{u}) e_{i j}(\mathbf{v}) .
$$

We shall omit from now on the variable $t$ in order to simplify the notations.

Using relations (2.5) and (2.6) and the boundary conditions on the various parts of $\Gamma$ :

$$
a(\mathbf{u}, \mathbf{v}-\dot{\mathbf{u}})-\int_{\Omega} \varphi_{1}(\mathbf{v}-\dot{\mathbf{u}})-\int_{\Gamma_{2}} \varphi_{2}(\mathbf{v}-\dot{\mathbf{u}})-\int_{\Gamma_{3}} \sigma_{\mathrm{N}}\left(v_{\mathrm{N}}-\dot{u}_{\mathrm{N}}\right)-\int_{\Gamma_{3}} \sigma_{\mathrm{T}}\left(\mathbf{v}_{\mathrm{T}}-\dot{\mathbf{u}}_{\mathrm{T}}\right)=0
$$

A variational formulation, equivalent to (2.9), of the Coulomb law is given by the following inequality (see [14]):

with

$$
\forall \mathbf{v} \in V \quad j(\mathbf{u}, \mathbf{v})-j(\mathbf{u}, \dot{\mathbf{u}})+\int_{\Gamma_{3}} \boldsymbol{\sigma}_{\mathrm{T}}\left(\mathbf{v}_{\mathrm{T}}-\dot{\mathbf{u}}_{\mathrm{T}}\right) \geq 0
$$

$$
j(\mathbf{u}, \mathbf{v})=\int_{\Gamma_{\lambda}} \mu\left|R \sigma_{\mathrm{N}}(\mathbf{u})\right|\left|\mathbf{v}_{\mathrm{T}}\right| .
$$

Then (2.11) and (2.12) yield the following implicit variational inequality:

$$
\forall \mathbf{v} \in V \quad a(\mathbf{u}, \mathbf{v}-\dot{\mathbf{u}})-(\mathbf{f}, \mathbf{v}-\dot{\mathbf{u}})+j(\mathbf{u}, \mathbf{v})-j(\mathbf{u}, \dot{\mathbf{u}})-\int_{\Gamma_{3}} \sigma_{\mathrm{N}}(\mathbf{u})\left(v_{\mathrm{N}}-\dot{u}_{\mathrm{N}}\right) \geq 0
$$

with

$$
(\mathbf{f}, \mathbf{v})=\int_{\Omega 2} \varphi_{1} \mathbf{v}+\int_{\Gamma_{2}} \varphi_{2} \mathbf{v}
$$

Concerning the unilateral condition (2.8) the following weak formulation can be given (if the solution is sufficiently regular):

$$
\forall \mathbf{z} \in K, \int_{\Gamma_{3}} \sigma_{\mathrm{N}}(\mathbf{u})\left(z_{\mathrm{N}}-u_{\mathrm{N}}\right) \geq 0
$$

We suppose that $\mu \in L^{\times}\left(\Gamma_{3}\right)$ and $\mu \geq 0$ almost everywhere on $\Gamma_{3}$, that $R: H^{-1 / 2}\left(\Gamma_{3}\right) \rightarrow L^{2}\left(\Gamma_{3}\right)$ is a compact linear operator, and that the initial condition $\mathbf{u}_{0}$ belongs to $\mathrm{K}$ and satisfies the following compatibility condition:

$$
a\left(\mathbf{u}_{0}, \mathbf{w}-\mathbf{u}_{0}\right)+j\left(\mathbf{u}_{0}, \mathbf{w}-\mathbf{u}_{0}\right) \geq\left(\mathbf{f}(0), \mathbf{w}-\mathbf{u}_{0}\right) \quad \forall \mathbf{w} \in K .
$$

We shall adopt the following weak formulation of problem $P_{1}$.

Problem $P_{2}$.

Find $\mathbf{u} \in W^{1.2}(0, T ; V)$ such that $\mathbf{u}(0)=\mathbf{u}_{0}$ and for almost all $t \in[0, T], \mathbf{u}(t) \in K$ and

$$
\begin{array}{cc}
a(\mathbf{u}(t), \mathbf{v}-\dot{\mathbf{u}}(t))+j(\mathbf{u}(t), \mathbf{v})-j(\mathbf{u}(t), \dot{\mathbf{u}}(t)) \geq(\mathbf{f}(t), \mathbf{v}-\dot{\mathbf{u}}(t))+\left\langle\sigma_{\mathrm{N}}(\mathbf{u}(t)), v_{\mathrm{N}}-\dot{u}_{\mathrm{N}}(t)\right\rangle & \forall \mathbf{v} \in \mathbf{V}, \\
\left\langle\sigma_{\mathrm{N}}(\mathbf{u}(t)), z_{\mathrm{N}}-u_{\mathrm{N}}(t)\right\rangle \geq 0 \quad \forall \mathbf{z} \in K . &
\end{array}
$$

If the solution $\mathbf{u}$ of problem $P_{2}$ is sufficiently regular, $\mathbf{u}$ shall also be a solution of problem $P_{1}$ because: 
-Equations (2.1)-(2.3) are easily obtained by choosing $\mathbf{v}=\dot{\mathbf{u}} \pm \boldsymbol{\varphi}$ on $\Omega$ with $\varphi \in\left(C_{0}^{x}(\Omega)\right)^{d}$ then equation (2.6) is obtained by setting $\mathbf{v}=\dot{\mathbf{u}} \pm \boldsymbol{\psi}$ on $\Gamma_{2}$ with $\boldsymbol{\psi} \in V$ and $\mathbf{v}=\dot{\mathbf{u}}$ on $\Gamma_{3}$.

-Inequality (2.12) then holds which establishes the friction relations (2.9).

-The choice of $\mathbf{u}$ in $K$ ensures the unilateral condition $u_{\mathrm{N}} \leq 0$ and by choosing $z_{\mathrm{N}}=0$ and $z_{N}=2 u_{N}$ in inequality $(2.14)$ we obtain:

$$
\int_{\Gamma_{3}} \sigma_{\mathrm{N}} u_{\mathrm{N}}=0
$$

It follows that $\int_{\Gamma_{7}} \sigma_{\mathrm{N}} z_{\mathrm{N}} \geq 0 \forall \mathbf{z} \in K$ which implies $\sigma_{\mathrm{N}} \leq 0$ and finally, using equation (2.18), that relation (2.8) holds.

\section{REMARK 2.1.}

It is interesting to note that we have $\left\langle\sigma_{\mathrm{N}}(\mathbf{u}(t)), \dot{u}_{\mathrm{N}}(t)\right\rangle=0$ for any element $\mathbf{u}$ such that, for all $t \in[0, T], \mathbf{u}(t)$ belongs to $K$ and satisfies inequality (2.17). This is obtained simply by using the fact that

$$
\left\langle\sigma_{\mathrm{N}}(\mathbf{u}(t)), \frac{u_{\mathrm{N}}(t+\Delta t)-u_{\mathrm{N}}(t)}{\Delta t}\right\rangle \geq 0
$$

and

$$
\left\langle\sigma_{\mathrm{N}}(\mathbf{u}(t)), \frac{u_{\mathrm{N}}(t-\Delta t)-u_{\mathrm{N}}(t)}{-\Delta t}\right\rangle \leq 0
$$

for all $\Delta t>0$ and sufficiently small and for all $t$ belonging to $] 0, T[$.

\section{REMARK 2.2.}

We shall not consider the mapping $j(\cdot, \cdot)$ defined as in equation $(2.13)$ but we shall define $j(\cdot, \cdot)$ by:

$$
j(\mathbf{u}, \mathbf{v})=\int_{\Gamma_{3}} \mu\left|R \sigma_{\mathrm{N}}(P \mathbf{u})\right|\left|\mathbf{v}_{\mathrm{T}}\right| \quad \text { for }(\mathbf{u}, \mathbf{v}) \in V \times V,
$$

where $P$ is the projection of $V$ onto the space $V_{0}$ with $V_{0}=\left\{\mathbf{w} \in V\right.$, div $\sigma(\mathbf{w})=-\boldsymbol{\varphi}_{1}$ in $\left.\Omega\right\}$. If $\mathbf{u}$ is a solution of $\mathrm{P}_{2}$ then $\mathbf{u}=P \mathbf{u}$ therefore both definitions coincide. This definition has the advantage of enabling us to define $j(\cdot, \cdot)$ on $V \times V[15]$.

\section{INCREMENTAL FORMULATION}

We shall prove the existence of a solution to the quasistatic problem by considering its variational formulation $P_{2}$ given in Section 2. The bilinear form $a(\cdot, \cdot)$ and the mapping $j(\cdot, \cdot)$ involved in this variational formulation have the following properties:

$a(\cdot, \cdot)$ is continuous on $V \times V$ and coercive, i.e. satisfies

$$
\begin{gathered}
\exists M>0 \quad \forall \mathbf{u} \in V \quad \forall \mathbf{v} \in V \quad a(\mathbf{u}, \mathbf{v}) \leq M\|\mathbf{u}\|\|\mathbf{v}\|, \\
\exists m>0 \quad \forall \mathbf{u} \in V \quad a(\mathbf{u}, \mathbf{u}) \geq m\|\mathbf{u}\|^{2},
\end{gathered}
$$

and the mapping $j(\cdot, \cdot)$ satisfies the following property (see [15]):

$$
\begin{aligned}
\exists C>0 \quad \forall\left(\mathbf{u}_{1}, \mathbf{u}_{2}, \mathbf{v}_{1}, \mathbf{v}_{2}\right) \in V^{4} \\
\left|j\left(\mathbf{u}_{1}, \mathbf{v}_{1}\right)-j\left(\mathbf{u}_{1}, \mathbf{v}_{2}\right)-j\left(\mathbf{u}_{2}, \mathbf{v}_{1}\right)+j\left(\mathbf{u}_{2}, \mathbf{v}_{2}\right)\right| \leq \bar{\mu} C\left\|\mathbf{u}_{1}-\mathbf{u}_{2}\right\|\left\|\mathbf{v}_{1}-\mathbf{v}_{2}\right\|
\end{aligned}
$$

with $\bar{\mu}=|\mu|_{L^{*}\left(\Gamma_{3}\right)} \cdot$ 
It shall be useful in the following to note that $j(\cdot, \cdot)$ also satisfies the following inequality:

$$
\forall\left(\mathbf{u}, \mathbf{v}_{1}, \mathbf{v}_{2}\right) \in V \quad j\left(\mathbf{u}, \mathbf{v}_{1}\right)-j\left(\mathbf{u}, \mathbf{v}_{2}\right) \leq j\left(\mathbf{u}, \mathbf{v}_{1}-\mathbf{v}_{2}\right) .
$$

An incremental formulation is obtained by operating a time discretization of problem $P_{2}$, taking $n \in \mathbf{N}^{*}$ and setting $\Delta t=T / n, t_{i}=i \cdot \Delta t$ and $\mathbf{f}^{i}=\mathbf{f}\left(t_{i}\right)$ for $i=0, \ldots, n$. We use an implicit scheme and obtain the following sequence $\left(P_{i}^{n}\right) i=0, \ldots, n-1$ of variational inequalities defined for a given $\mathbf{u}^{0} \in K$ by:

Problem $P_{i}^{n}$.

$\left\{\begin{aligned} \text { Find } \mathbf{u}^{i+1} \in K \text { such that } & \begin{array}{rl}a\left(\mathbf{u}^{i+1}, \mathbf{v}-\frac{\mathbf{u}^{i+1}-\mathbf{u}^{i}}{\Delta t}\right)+j\left(\mathbf{u}^{i+1}, \mathbf{v}\right)-j\left(\mathbf{u}^{i+1}, \frac{\mathbf{u}^{i+1}-\mathbf{u}^{i}}{\Delta t}\right) \geq & \left(\mathbf{f}^{i+1}, \mathbf{v}-\frac{\mathbf{u}^{i+1}-\mathbf{u}^{i}}{\Delta t}\right) \\ & +\left\langle\sigma_{\mathrm{N}}\left(\mathbf{u}^{i+1}\right), v_{\mathrm{N}}-\frac{u_{\mathrm{N}}^{i+1}-u_{\mathrm{N}}^{i}}{\Delta t}\right\rangle \quad \forall \mathbf{v} \in V, \\ \left\langle\sigma_{\mathrm{N}}\left(\mathbf{u}^{i+1}\right), z_{\mathrm{N}}-u_{\mathrm{N}}^{i+1}\right\rangle \geq 0 \quad \forall \mathbf{z} \in K . & \end{array}\end{aligned}\right.$

\subsection{Existence of a solution of problem $P_{i}^{n}$}

In order to show that there exists a solution of each problem $P_{i}^{n}$ we shall construct equivalent problems.

Let us first consider the following sequence of problems, obviously equivalent to $P_{i}^{n}$, obtained by multiplying the first inequality in $P_{i}^{n}$ by $\Delta t$ and by setting $\mathbf{w}=\Delta t \mathbf{v}+\mathbf{u}^{i}$

Problem $Q_{i}^{n}$.

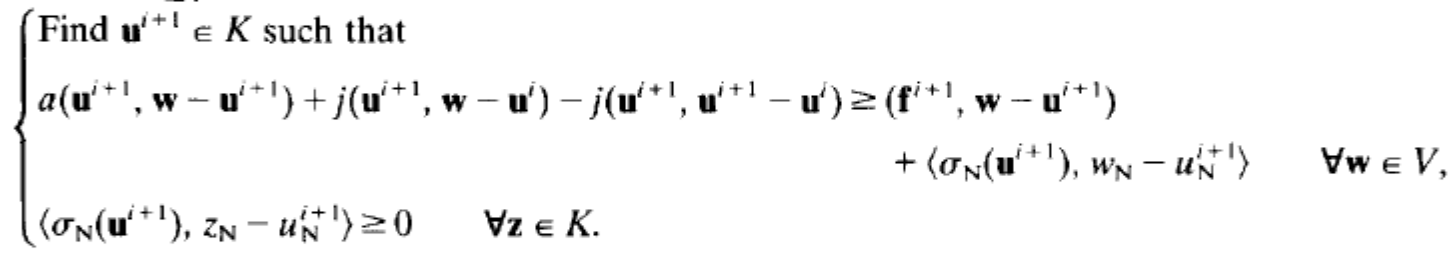

Problems $Q_{i}^{n}$ are a sequence of intermediate problems which we are going to show to be equivalent to the following sequence:

Problem $S_{i}^{n}$.

Find $\mathbf{u}^{i+1} \in K$ such that

$\left\{a\left(\mathbf{u}^{i+1}, \mathbf{w}-\mathbf{u}^{i+1}\right)+j\left(\mathbf{u}^{i+1}, \mathbf{w}-\mathbf{u}^{i}\right)-j\left(\mathbf{u}^{i+1}, \mathbf{u}^{i+1}-\mathbf{u}^{i}\right) \geq\left(\mathbf{f}^{i+1}, \mathbf{w}-\mathbf{u}^{i+1}\right) \quad \forall \mathbf{w} \in K\right.$.

REMARK 3.1.

Inequalities such as $S_{i}^{n}$ have been shown to possess a unique solution if the friction coefficient which appears in $j(\cdot, \cdot)$ is sufficiently small (see, e.g. $[10,11,15]$ ). To be more precise the friction coefficient must satisfy the following inequality $\bar{\mu} \leq \mu_{1}<m / C$ where $m$ and $C$ have been defined by the relations (3.2) and (3.3). We shall from now on suppose that the friction coefficient is small enough to ensure that the above inequality is true.

By a similar argument to that used in Section 2, $S_{i}^{n}$ is shown to be equivalent to the following relations:

$$
\begin{gathered}
\operatorname{div} \sigma\left(\mathbf{u}^{i+1}\right)+\varphi_{1}^{i+1}=0 \text { in } \Omega \\
\sigma_{\mathrm{N}}\left(\mathbf{u}^{i+1}\right) \cdot \mathbf{n}=\varphi_{2}^{i+1} \text { on } \Gamma_{2} \\
\left|\boldsymbol{\sigma}_{\mathrm{T}}\left(\mathbf{u}^{i+1}\right)\right| \leq \mu\left|R\left(\sigma_{\mathrm{N}}\left(\mathbf{u}^{i+1}\right)\right)\right| \text { on } \Gamma_{3}
\end{gathered} \text { and } \begin{aligned}
& \left\{\begin{array}{l}
\text { if }\left|\boldsymbol{\sigma}_{\mathrm{T}}\left(\mathbf{u}^{i+1}\right)\right|<\mu \\
\text { if not then } \left.\mathbf{u}_{\mathrm{T}}^{i+1}-\mathbf{u}_{\mathrm{T}}^{i}=-\lambda\left(\sigma_{\mathrm{N}}\left(\mathbf{u}_{\mathrm{T}}^{i+1}\right)\right) \mid \text { then } \mathbf{u}_{\mathrm{T}}^{i+1}=\mathbf{u}_{\mathrm{T}}^{i+1}\right) \lambda \geq 0
\end{array}\right. \\
& u_{\mathrm{N}}^{i+1} \leq 0 \quad \sigma_{\mathrm{N}}\left(\mathbf{u}^{i+1}\right) \leq 0 \quad \sigma_{\mathrm{N}}\left(\mathbf{u}^{i+1}\right) u_{\mathrm{N}}^{i+1}=0 \text { on } \Gamma_{3} .
\end{aligned}
$$

It is also easy to verify that above conditions are equivalent to $Q_{i}^{n}$. 


\section{ReMARK 3.2.}

We suppose that $\mathbf{u}^{\circ}$ satisfies the same condition (2.15) that we have imposed on $\mathbf{u}_{0}$, i.e. that $\mathbf{u}^{0}$ satisfies:

$$
a\left(\mathbf{u}^{0}, \mathbf{w}-\mathbf{u}^{0}\right)+j\left(\mathbf{u}^{0}, \mathbf{w}-\mathbf{u}^{0}\right) \geq\left(\mathbf{f}^{0}, \mathbf{w}-\mathbf{u}^{0}\right) \quad \forall \mathbf{w} \in K .
$$

Then by setting $\mathbf{w}=\mathbf{u}^{0}$ in $S_{0}^{n}$ and $\mathbf{w}=\mathbf{u}^{1}$ in inequality (3.5) and adding both inequalities we obtain

$$
-a\left(\mathbf{u}^{1}-\mathbf{u}^{0}, \mathbf{u}^{1}-\mathbf{u}^{0}\right)+j\left(\mathbf{u}^{0}, \mathbf{u}^{1}-\mathbf{u}^{0}\right)-j\left(\mathbf{u}^{1}, \mathbf{u}^{1}-\mathbf{u}^{0}\right) \geq\left(\mathbf{f}^{1}-\mathbf{f}^{0}, \mathbf{u}^{1}-\mathbf{u}^{0}\right)
$$

as $a(\cdot, \cdot)$ and $j(\cdot, \cdot)$ satisfy $(3.2)$ and $(3.3),(3.6)$ yields

$$
\begin{gathered}
m\left\|\mathbf{u}^{1}-\mathbf{u}^{0}\right\|^{2} \leq\left\|\mathbf{f}^{1}-\mathbf{f}^{0}\right\|\left\|\mathbf{u}^{1}-\mathbf{u}^{0}\right\|+\bar{\mu} C\left\|\mathbf{u}^{1}-\mathbf{u}^{0}\right\|^{2} \\
(m-\bar{\mu} C)\left\|\mathbf{u}^{1} \mathbf{u}^{0}\right\| \leq\left\|\mathbf{f}^{1}-\mathbf{f}^{0}\right\| .
\end{gathered}
$$

Therefore if $\bar{\mu} C<m, \mathbf{f}^{1}=\mathbf{f}^{0}$ implies that $\mathbf{u}^{1}=\mathbf{u}^{0}$.

In the following we shall suppose that $\mathbf{u}^{0}$ satisfies inequality (3.5).

\subsection{Bounds for the solution of $P_{i}^{n}$}

In this section we shall exhibit bounds for the incremental solution $\mathbf{u}^{i}$ and also for the difference $\mathbf{u}^{i+1}-\mathbf{u}^{i}$.

By setting in inequalities $S_{i}^{n}$, for $i=0, \ldots, n-1, \mathbf{w}=\mathbf{0}$ we obtain:

$$
a\left(\mathbf{u}^{i+1}, \mathbf{u}^{i+1}\right) \leq\left(\mathbf{f}^{i+1}, \mathbf{u}^{i+1}\right)+j\left(\mathbf{u}^{i+1}-\mathbf{u}^{i}\right)-j\left(\mathbf{u}^{i+1}, \mathbf{u}^{i+1}-\mathbf{u}^{i}\right) \quad \forall i=0, \ldots, n-1
$$

and by (3.4) we have

$$
a\left(\mathbf{u}^{i+1}, \mathbf{u}^{i+1}\right) \leq\left(\mathbf{f}^{i+1}, \mathbf{u}^{i+1}\right)+j\left(\mathbf{u}^{i+1},-\mathbf{u}^{i+1}\right) \quad \forall i=0, \ldots, n-1 .
$$

From relations (3.2) and (3.3) we obtain:

$$
m\left\|\mathbf{u}^{i+1}\right\|^{2} \leq\left\|\mathbf{f}^{i+1}\right\|\left\|\mathbf{u}^{i+1}\right\|+\bar{\mu} C\left\|\mathbf{u}^{i+1}\right\|^{2} \quad \forall i=0, \ldots, n-1,
$$

so that if $\bar{\mu} C<m$ we have:

$$
\left\|\mathbf{u}^{i+1}\right\| \leq \frac{\left\|\mathbf{f}^{i+1}\right\|}{m-\ddot{\mu} C} \quad \forall i=0, \ldots, n-1,
$$

and by setting $\mathbf{w}=\mathbf{0}$ in condition (3.5) we finally obtain:

$$
\left\|\mathbf{u}^{i}\right\| \leq \frac{\left\|\mathbf{f}^{i}\right\|}{m-\bar{\mu} C} \quad \forall i=0, \ldots, n .
$$

Let us now set $\Delta \mathbf{u}^{i}=\mathbf{u}^{i+1}-\mathbf{u}^{i}$ and $\Delta \mathbf{f}^{i}=\mathbf{f}^{i+1}-\mathbf{f}^{i}$ for $i=0, \ldots, n-1$. Considering inequality $S_{i}^{n}$ in which we set $\mathbf{w}=\mathbf{u}^{i}$ and adding it to inequality $S_{i-1}^{n}$ in which we set $\mathbf{w}=\mathbf{u}^{i+1}$ [if $i=0$ we set $\mathbf{w}=\mathbf{u}^{\prime}$ in condition (3.5)] we obtain the following inequality:

$$
a\left(\Delta \mathbf{u}^{i}, \Delta \mathbf{u}^{i}\right) \leq\left(\Delta \mathbf{f}^{i}, \Delta \mathbf{u}^{i}\right)+j\left(\mathbf{u}^{i}, \Delta \mathbf{u}^{i}+\Delta \mathbf{u}^{i-1}\right)-j\left(\mathbf{u}^{i}, \Delta \mathbf{u}^{i-1}\right)-j\left(\mathbf{u}^{i+1}, \Delta \mathbf{u}^{i}\right) \quad \forall i=0, \ldots, n-1 .
$$

And from relations (3.2) and (3.3) we obtain the following bound for $\Delta \mathbf{u}^{i}$ :

$$
\left\|\Delta \mathbf{u}^{i}\right\| \leq \frac{C^{i}\left\|\Delta \mathbf{f}^{i}\right\|}{m-\bar{\mu} C} \quad \forall i=0, \ldots, n-1 .
$$




\section{EXISTENCE OF A QUASISTATIC SOLUTION}

We define, for all $n \in \mathbf{N}^{*}$, a function $\mathbf{f}_{n}:[0, T] \rightarrow V$ by $\mathbf{f}_{n}(0)=\mathbf{f}(0)$ and $\mathbf{f}_{n}(t)=\mathbf{f}\left(t_{i+1}\right)$ for $\left.t \in] t_{i}, t_{i+1}\right]$ where $t_{i}=i \cdot \Delta t$ and $\Delta t=T / n$.

We then consider inequalities $S_{i}^{n}$ with $\mathbf{f}^{i}=\mathbf{f}\left(t_{i}\right)$ for $i=0, \ldots, n$ in order to define a function $\mathbf{u}_{n} \in L^{2}(0, T ; V)$ by $\mathbf{u}_{n}(0)=\mathbf{u}^{0}=\mathbf{u}_{0}$ where $\mathbf{u}_{0} \in K$ is the initial condition of problem $P_{2}$ satisfying (2.15) and for $\left.t \in] t_{i}, t_{i+1}\right]$ by $\mathbf{u}_{n}(t)=\mathbf{u}^{i+1}$ solution of $S_{i}^{n}, i=0, \ldots, n-1$.

\subsection{Weak convergence for $\boldsymbol{u}_{n}$}

Four quite straightforward results can be found in the following lemma and shall be useful tools in the proofs of the next lemmas.

LEMMA 1.

$\mid \begin{aligned} & \exists C_{0} \text { such that } \forall t \in[0, T], \forall n \in \mathbf{N}^{*}\left\|\mathbf{u}_{n}(t)\right\| \leq C_{0}, \\ & \exists C_{1} \text { such that } \forall n \in \mathbf{N}^{*} \forall i=0, \ldots, n-1,\left\|\mathbf{u}^{i+1}-\mathbf{u}^{i}\right\| \leq C_{1} \int_{t_{i}}^{t_{i+1}}\|\dot{\mathbf{f}}(\tau)\| \mathrm{d} \tau, \\ & \exists C_{1} \text { such that } \forall n \in \mathbf{N}^{*} \forall i=0, \ldots, n-1,\left\|\mathbf{u}^{i+1}-\mathbf{u}^{i}\right\|^{2} \leq C_{1}^{2} \Delta t \int_{t_{i}}^{t_{i+1}}\|\dot{\mathbf{f}}(\tau)\|^{2} \mathrm{~d} \tau, \\ & \exists C_{1} \text { such that } \forall n \in \mathbf{N}^{*} \forall s, t \in[0, T], s<t,\left\|\mathbf{u}_{n}(s)-\mathbf{u}_{n}(t)\right\| \leq C_{1} \int_{s}^{\min (t+\Delta, T)}\|\dot{\mathbf{f}}(\tau)\| \mathrm{d} \tau .\end{aligned}$

Proof.

Inequality (4.1) follows directly from (3.7) and inequality (4.2) follows from (3.8) and from the fact that $\mathbf{f}$ is absolutely continuous (see [13]). Inequality (4.3) is established by using (4.2) and the Cauchy-Schwarz inequality.

In order to establish (4.4) for $s<t$ and for a given $n$ let us take $i \leq j$ such that $\left.s \in] t_{i}, t_{i+1}\right]$ and $\left.t \in] t_{j}, t_{j+1}\right]$ so that

$$
\left\|\mathbf{u}_{n}(t)-\mathbf{u}_{n}(s)\right\|=\left\|\mathbf{u}^{j+1}-\mathbf{u}^{i+1}\right\| \leq \sum_{m=i+1}^{j}\left\|\mathbf{u}^{m+1}-\mathbf{u}^{m}\right\|
$$

so that from inequality (4.2) we have

$$
\left\|\mathbf{u}_{n}(t)-\mathbf{u}_{n}(s)\right\| \leq C_{1} \sum_{m=i+1}^{j} \int_{t_{m}}^{t_{m+1}}\|\dot{\mathbf{f}}(\tau)\| \mathrm{d} \tau=C_{1} \int_{t_{i+1}}^{t_{j+1}}\|\dot{\mathbf{f}}(\tau)\| \mathrm{d} \tau \leq C_{1} \int_{s}^{\min (t+\Delta t, T)}\|\dot{\mathbf{f}}(\tau)\| \mathrm{d} \tau .
$$

LEMMA 2.

There exists a subsequence $\left(\mathbf{u}_{n_{p}}\right)_{p}$ of $\mathbf{u}_{n}$ such that for all $t \in[0, T]\left(\mathbf{u}_{n_{p}}(t)\right)_{p}$ converges weakly in $V$.

ProOF.

Let $E$ be a countable dense subset of $[0, T], E=\left(\tau_{j}\right)_{j \in \mathbf{N}}$. As by (4.1), for all $j \in \mathbf{N}$ and for all $n \in \mathbf{N}^{*}$, we have $\left\|\mathbf{u}_{n}\left(\tau_{j}\right)\right\| \leq C_{0}$, by a diagonal process we can extract a subsequence $\left(\mathbf{u}_{n_{p}}\right)_{p}$ of $\mathbf{u}_{n}$ such that for all $j \in \mathbf{N}$ the sequence $\left(\mathbf{u}_{n_{p}}\left(\tau_{j}\right)\right)_{p}$ converges weakly towards an element of $V$ noted by $\mathbf{u}\left(\tau_{j}\right)$. We shall omit the subscript $p$ from now on whenever no confusion is possible.

Let us now consider for all $\phi \in V$ and for all $t \in\left[0, T\left[\right.\right.$ the sequence $\left(\mathbf{u}_{n}(t), \boldsymbol{\phi}\right)$ for which we have

$$
\begin{aligned}
& \forall q \geq 0 \text { and } \forall \tau_{j} \\
& \left|\left(\mathbf{u}_{n+q}(t)-\mathbf{u}_{n}(t), \boldsymbol{\phi}\right)\right| \leq\left|\left(\mathbf{u}_{n+q}(t)-\mathbf{u}_{n+q}\left(\tau_{j}\right), \boldsymbol{\phi}\right)\right|+\left|\left(\mathbf{u}_{n+q}\left(\tau_{j}\right)-\mathbf{u}_{n}\left(\tau_{j}\right), \boldsymbol{\phi}\right)\right|+\left|\left(\mathbf{u}_{n}\left(\tau_{j}\right)-\mathbf{u}_{n}(t), \boldsymbol{\phi}\right)\right| \\
& \left|\left(\mathbf{u}_{n+q}(t)-\mathbf{u}_{n}(\mathbf{t}), \boldsymbol{\phi}\right)\right| \leq\|\boldsymbol{\phi}\|\left(\left\|\mathbf{u}_{n+q}(t)-\mathbf{u}_{n+q}\left(\tau_{j}\right)\right\|+\left\|\mathbf{u}_{n}\left(\tau_{j}\right)-\mathbf{u}_{n}(t)\right\|\right)+\left|\left(\mathbf{u}_{n+q}\left(\tau_{j}\right)-\mathbf{u}_{n}\left(\tau_{j}\right), \boldsymbol{\phi}\right)\right|
\end{aligned}
$$


so that by taking $\tau_{j}>t$ and using relation (4.4) we obtain

$$
\left|\left(\mathbf{u}_{n+q}(t)-\mathbf{u}_{n}(t), \boldsymbol{\phi}\right)\right| \leq 2\|\boldsymbol{\phi}\| C_{1} \int_{t}^{\tau_{j}+(T / n)}\|\mathbf{f}(\tau)\| \mathrm{d} \tau+\left|\left(\mathbf{u}_{n+q}\left(\tau_{j}\right)-\mathbf{u}_{n}\left(\tau_{j}\right), \boldsymbol{\phi}\right)\right| .
$$

As $E$ is dense in $[0, T]$ there exists a $\tau_{j}$ arbitrarily close to $t$ and as the sequence $\left(\mathbf{u}_{n}\left(\tau_{j}\right), \boldsymbol{\phi}\right)$ is convergent therefore a Cauchy sequence, for all $t \in\left[0, T\left[,\left(\mathbf{u}_{n}(t), \boldsymbol{\phi}\right)\right.\right.$ is also a Cauchy sequence of $\mathbf{R}$ therefore convergent. The case $t=T$ can be treated by a similar argument.

\section{REMARK 4.1.}

For all $t$ belonging to $[0, T]$ and for all $n \in \mathbf{N}^{*}, \mathbf{u}_{n}(t)$ belongs to $K$ so that the weak limit $\mathbf{u}(t)$ also belongs to $K$.

In order to show that $\mathbf{u}$ is sufficiently regular we shall now define the following sequence of functions:

$$
\left\{\begin{array}{c}
\left.\left.\tilde{\mathbf{u}}_{n}(t)=\mathbf{u}^{i}+\frac{\left(t-t_{i}\right)}{\Delta t}\left(\mathbf{u}^{i+1}-\mathbf{u}^{i}\right) \quad t \in\right] t_{i}, t_{i+1}\right] \\
\tilde{\mathbf{u}}_{n}(0)=\mathbf{u}^{0},
\end{array}\right.
$$

where $n$ belongs to the subset of $\mathbf{N}$ corresponding to the subsequence of $\mathbf{u}_{n}$ one has exhibited in the proof of lemma 2 . The mapping $\tilde{\mathbf{u}}_{n}$ is obviously an element of $W^{1,2}(0, T ; V)$.

\section{Lemma 3.}

There exists a subsequence of $\tilde{\mathbf{u}}_{n}$ that converges weakly in $W^{1.2}(0, T ; V)$ and the weak limit of this subsequence is equal to $\mathbf{u}$ in $L^{2}(0, T ; V)$.

Proof.

The sequence $\tilde{\mathbf{u}}_{n}$ is bounded in $W$ indeed, by using relations (4.1) and (4.3),

$$
\begin{aligned}
\left\|\tilde{\mathbf{u}}_{n}\right\|_{W}^{2}= & \int_{0}^{T}\left\|\tilde{\mathbf{u}}_{n}(t)\right\|^{2} \mathrm{~d} t+\int_{0}^{T}\left\|\frac{\mathrm{d}}{\mathrm{d} t} \tilde{\mathbf{u}}_{n}(t)\right\|^{2} \mathrm{~d} t \\
& \leq C_{0}^{2} T+\sum_{i=0}^{n-1} \int_{t_{i}}^{t_{i+1}}\left\|\frac{\mathbf{u}^{i+1}-\mathbf{u}^{i}}{\Delta t}\right\|^{2} \mathrm{~d} t \\
& \leq C_{0}^{2} T+\sum_{i=0}^{n-1} \int_{t_{i}}^{t_{i+1}} \frac{C_{1}^{2}}{\Delta t}\left(\int_{t_{i}}^{t_{i-1}}\|\mathbf{f}(\tau)\|^{2} \mathrm{~d} \tau\right) \mathrm{d} t
\end{aligned}
$$

so that $\left\|\tilde{\mathbf{u}}_{n}\right\|_{w}^{2} \leq C_{0}^{2} T+C_{1}^{2} \int_{0}^{T}\|\dot{\mathbf{f}}(t)\|^{2} \mathrm{~d} t<+\infty$.

There exists therefore a subsequence of $\tilde{\mathbf{u}}_{n}$ which converges weakly in $W^{1.2}(0, T ; V)$ towards

$\tilde{\mathbf{u}}$. Let us now consider that $n$ belongs to the subset of $\mathbf{N}$ corresponding to this subsequence.

We shall now show that $\mathbf{u}=\tilde{\mathbf{u}}$. We have

$$
\begin{aligned}
\left|\left(\left(\tilde{\mathbf{u}}_{n}-\mathbf{u}_{n}, \boldsymbol{\varphi}\right)\right)\right| & =\left|\int_{0}^{T}\left(\tilde{\mathbf{u}}_{n}(t)-\mathbf{u}_{n}(t), \boldsymbol{\varphi}(t)\right) \mathrm{d} t\right|=\sum_{i=0}^{n-1} \int_{t_{i}}^{t_{i+1}}\left(\mathbf{u}^{i}+\frac{t-t_{i}}{\Delta t}\left(\mathbf{u}^{i+1}-\mathbf{u}^{i}\right)-\mathbf{u}^{i+1}, \boldsymbol{\varphi}(t)\right) \mathrm{d} t \\
\leq & \sum_{i=0}^{n-1} \int_{t_{i}}^{t_{i+1}}\left\|\mathbf{u}^{i+1}-\mathbf{u}^{i}\right\|\|\boldsymbol{\varphi}(t)\| \mathrm{d} t \leq\left(\sum_{i=0}^{n-1} \int_{t_{i}}^{t_{i+1}}\left\|\mathbf{u}^{i+1}-\mathbf{u}^{i}\right\|^{2} \mathrm{~d} t\right)^{1 / 2}\left(\sum_{i=0}^{n-1} \int_{t_{i}}^{t_{i+1}}\|\boldsymbol{\varphi}(t)\|^{2} \mathrm{~d} t\right)^{1 / 2} .
\end{aligned}
$$

From inequality (4.3) it follows that

$$
\left|\left(\left(\tilde{\mathbf{u}}_{n}-\mathbf{u}_{n}, \boldsymbol{\varphi}\right)\right)\right| \leq C_{1} \Delta t\left(\int_{0}^{T}\|\dot{\mathbf{f}}(t)\|^{2} \mathrm{~d} t\right)^{1 / 2}\left(\int_{0}^{T}\|\boldsymbol{\varphi}(t)\|^{2} \mathrm{~d} t\right)^{1 / 2} .
$$

So that $\left(\left(\tilde{\mathbf{u}}_{n}-\mathbf{u}_{n}, \varphi\right)\right) \underset{n \rightarrow \infty}{\longrightarrow} 0 \quad \forall \varphi \in L^{2}(0, T ; V)$. 
And as for all $t \in[0, T], \mathbf{u}_{n}(t)$ converges weakly towards $\mathbf{u}(t)$, we shall have

$$
\left(\left(\mathbf{u}_{n}-\mathbf{u}, \varphi\right)\right) \underset{n \rightarrow x}{\longrightarrow} 0 \text { and therefore }((\tilde{\mathbf{u}}-\mathbf{u}, \varphi))=0 \quad \forall \varphi \in L^{2}(0, T ; V) .
$$

Thus as $\tilde{\mathbf{u}}$ belongs to $W^{1,2}(0, T ; V)$ we shall also have $(\mathrm{d} / \mathrm{d} t) \tilde{\mathbf{u}}_{n}$ converging weakly towards

$$
\frac{\mathrm{d}}{\mathrm{d} t} \tilde{\mathbf{u}}=\frac{\mathrm{d}}{\mathrm{d} t} \mathbf{u} \text { in } L^{2}(0, T ; V) .
$$

\subsection{The weak limit of $\boldsymbol{u}_{n}$ is a solution of problem $P_{2}$}

The functions $\mathbf{u}_{n}$ and $\tilde{\mathbf{u}}_{n}$ are defined by using $\mathbf{u}^{i+1}$, solution of $S_{i}^{n}$, but also solution of $P_{i}^{n}$, for $i=0, \ldots, n-1$. A solution of $P_{i}^{n}$ is such that $\left\langle\sigma_{\mathrm{N}}\left(\mathbf{u}^{i+1}\right), u_{\mathrm{N}}^{i+1}\right\rangle=0$ and as $\sigma_{\mathrm{N}}\left(\mathbf{u}^{i+1}\right) \leq 0$ and $u_{\mathrm{N}}^{i} \leq 0$ we have $\left\langle\sigma_{\mathrm{N}}\left(\mathbf{u}^{i+1}\right), u_{\mathrm{N}}^{i}\right\rangle \geq 0$ so that

$$
\left\langle\sigma_{\mathrm{N}}\left(\mathbf{u}^{i+1}\right), v_{\mathrm{N}}-\frac{u_{\mathrm{N}}^{i+1}-u_{\mathrm{N}}^{i}}{\Delta t}\right\rangle \geq\left\langle\sigma_{\mathrm{N}}\left(\mathbf{u}^{i+1}\right), v_{\mathrm{N}}\right\rangle
$$

and therefore $\mathbf{u}^{i+1}$ shall satisfy for $i=0, \ldots, n-1$ :

$$
a\left(\mathbf{u}^{i+1}, \mathbf{v}-\frac{\mathbf{u}^{i+1}-\mathbf{u}^{i}}{\Delta t}\right)+j\left(\mathbf{u}^{i+1}, \mathbf{v}\right)-j\left(\mathbf{u}^{i+1}, \frac{\boldsymbol{u}^{i+1}-\mathbf{u}^{i}}{\Delta t}\right) \geq\left(\mathbf{f}^{i+1}, \mathbf{v}-\frac{\mathbf{u}^{i+1}-\mathbf{u}^{i}}{\Delta t}\right)+\left\langle\sigma_{\mathrm{N}}\left(\mathbf{u}^{i+1}\right), v_{\mathrm{N}}\right\rangle .
$$

So that we have for all $\mathbf{v} \in L^{2}(0, T ; V)$

$$
\begin{aligned}
a\left(\mathbf{u}_{n}(t), \mathbf{v}(t)-\frac{\mathrm{d}}{\mathrm{d} t} \tilde{\mathbf{u}}_{n}(t)\right)+j\left(\mathbf{u}_{n}(t), \mathbf{v}(t)\right)-j\left(\mathbf{u}_{n}(t), \frac{\mathrm{d}}{\mathrm{d} t} \tilde{\mathbf{u}}_{n}(t)\right) \geq & \left(\mathbf{f}_{n}(t), \mathbf{v}(t)-\frac{\mathrm{d}}{\mathrm{d} t} \tilde{\mathbf{u}}_{n}(t)\right) \\
& +\left\langle\sigma_{\mathrm{N}}\left(\mathbf{u}_{n}(t)\right), v_{\mathrm{N}}(\mathrm{t})\right\rangle
\end{aligned}
$$

and by integrating both sides of this inequality on $[0, T]$ we obtain:

$$
\begin{aligned}
& \int_{0}^{T}\left(a\left(\mathbf{u}_{n}(t), \mathbf{v}(t)-\frac{\mathrm{d}}{\mathrm{d} t} \tilde{\mathbf{u}}_{n}(t)\right)+j\left(\mathbf{u}_{n}(t), \mathbf{v}(t)\right)-j\left(\mathbf{u}_{n}(t), \frac{\mathrm{d}}{\mathrm{d} t} \tilde{\mathbf{u}}_{n}(t)\right)\right) \mathrm{d} t \\
& \quad \geq \int_{0}^{T}\left(\mathbf{f}_{n}(t), \mathbf{v}(t)-\frac{\mathrm{d}}{\mathrm{d} t} \tilde{\mathbf{u}}_{n}(t)\right) \mathrm{d} t+\int_{0}^{T}\left\langle\sigma_{\mathrm{N}}\left(\mathbf{u}_{n}(t)\right), v_{\mathrm{N}}(t)\right\rangle \mathrm{d} t .
\end{aligned}
$$

LEMMA 4.

For all $\mathbf{v} \in L^{2}(0, T ; V)$ we have

$$
\begin{aligned}
& \lim _{n \rightarrow \infty} \int_{0}^{T}\left(\mathbf{f}_{n}(t), \mathbf{v}(t)-\frac{\mathrm{d}}{\mathrm{d} t} \tilde{\mathbf{u}}_{n}(t)\right) \mathrm{d} t=\int_{0}^{T}(\mathbf{f}(t), \mathbf{v}(t)-\dot{\mathbf{u}}(t)) \mathrm{d} t, \\
& \lim _{n \rightarrow \infty} \int_{0}^{T} j\left(\mathbf{u}_{n}(t), \mathbf{v}(t)\right) \mathrm{d} t=\int_{0}^{T} j(\mathbf{u}(t), \mathbf{v}(t)) \mathrm{d} t, \\
& \lim _{n \rightarrow \infty} \int_{0}^{T} a\left(\mathbf{u}_{n}(t), \mathbf{v}(t)\right) \mathrm{d} t=\int_{0}^{T} a(\mathbf{u}(t), \mathbf{v}(t)) \mathrm{d} t .
\end{aligned}
$$

\section{Proof.}

The proof is straightforward as $\mathbf{u}_{n}$ converges weakly towards $\mathbf{u},(\mathrm{d} / \mathrm{d} t) \tilde{\mathbf{u}}_{n}$ converges weakly towards $\mathbf{u}$ in $L^{2}(0, T ; V)$ and $\mathbf{f}_{n}$ converges strongly towards $\mathbf{f}$.

\section{Lemma 5.}

$$
\mid \begin{aligned}
& \text { We have } \\
& \liminf _{n \rightarrow \infty} \int_{0}^{T} a\left(\mathbf{u}_{n}(t), \frac{\mathrm{d}}{\mathrm{d} t} \tilde{\mathbf{u}}_{n}(t)\right) \mathrm{d} t \geq \int_{0}^{T} a(\mathbf{u}(t), \dot{\mathbf{u}}(t)) \mathrm{d} t .
\end{aligned}
$$


Proof.

By using the fact that the mapping $a(\cdot, \cdot)$ is bilinear, symmetric and coercive,

$$
\begin{gathered}
\int_{0}^{T} a\left(\mathbf{u}_{n}(t), \frac{\mathrm{d}}{\mathrm{d} t} \tilde{\mathbf{u}}_{n}(t)\right) \mathrm{d} t=\sum_{i=0}^{n-1} \frac{1}{\Delta t} \int_{t_{i}}^{t_{i+1}} a\left(\mathbf{u}^{i+1}, \mathbf{u}^{i+1}-\mathbf{u}^{i}\right) \mathrm{d} t=\sum_{i=0}^{n-1} a\left(\mathbf{u}^{i+1}, \mathbf{u}^{i+1}-\mathbf{u}^{i}\right) \\
\int_{0}^{T} a\left(\mathbf{u}_{n}(t), \frac{\mathrm{d}}{\mathrm{d} t} \tilde{\mathbf{u}}_{n}(t)\right) \mathrm{d} t \geq \sum_{i=0}^{n-1} a\left(\mathbf{u}^{i+1}, \mathbf{u}^{i+1}-\mathbf{u}^{i}\right)-\frac{1}{2} \sum_{i=0}^{n-1} a\left(\mathbf{u}^{i+1}-\mathbf{u}^{i}, \mathbf{u}^{i+1}-\mathbf{u}^{i}\right)
\end{gathered}
$$

and

$\sum_{i=0}^{n-1} a\left(\mathbf{u}^{i+1}, \mathbf{u}^{i+1}-\mathbf{u}^{i}\right)-\frac{1}{2} \sum_{i=0}^{n-1} a\left(\mathbf{u}^{i+1}-\mathbf{u}^{i}, \mathbf{u}^{i+1}-\mathbf{u}^{i}\right)=\frac{1}{2} \sum_{i=0}^{n-1}\left[a\left(\mathbf{u}^{i+1}, \mathbf{u}^{i+1}-\mathbf{u}^{i}\right)+a\left(\mathbf{u}^{i+1}-\mathbf{u}^{i}, \mathbf{u}^{i}\right)\right]$

$$
=\frac{1}{2} \sum_{i=0}^{n-1}\left[a\left(\mathbf{u}^{i+1}, \mathbf{u}^{i+1}\right)-a\left(\mathbf{u}^{i}, \mathbf{u}^{i}\right)\right]=\frac{1}{2}\left[a\left(\mathbf{u}^{n}, \mathbf{u}^{n}\right)-a\left(\mathbf{u}^{0}, \mathbf{u}^{0}\right)\right]
$$

so that

$$
\int_{0}^{T} a\left(\mathbf{u}_{n}(t), \frac{\mathrm{d}}{\mathrm{d} t} \tilde{\mathbf{u}}_{n}(t)\right) \mathrm{d} t \geq \frac{1}{2}\left[a\left(\mathbf{u}_{n}(T), \mathbf{u}_{n}(T)\right)-a\left(\mathbf{u}_{n}(0), \mathbf{u}_{n}(0)\right)\right]=\frac{1}{2}\left[a\left(\mathbf{u}_{n}(T), \mathbf{u}_{n}(T)\right)-a\left(\mathbf{u}_{0}, \mathbf{u}_{0}\right)\right]
$$

and as we have

$$
\int_{0}^{T} a(\mathbf{u}(t), \dot{\mathbf{u}}(t)) \mathrm{d} t=\frac{1}{2} \int_{0}^{T} \frac{\mathrm{d}}{\mathrm{d} t} a(\mathbf{u}(t), \mathbf{u}(t)) \mathrm{d} t=\frac{1}{2}[a(\mathbf{u}(T), \mathbf{u}(T))-a(\mathbf{u}(0), \mathbf{u}(0))],
$$

finally we obtain

$\int_{0}^{T} a(\mathbf{u}(t), \mathbf{u}(t)) \mathrm{d} t \leq \liminf _{n \rightarrow \infty} \frac{1}{2}\left[a\left(\mathbf{u}_{n}(T), \mathbf{u}_{n}(T)\right)-a\left(\mathbf{u}_{n}(0), \mathbf{u}_{n}(0)\right)\right] \leq \liminf _{n \rightarrow \infty} \int_{0}^{T} a\left(\mathbf{u}_{n}(t), \frac{\mathrm{d}}{\mathrm{d} t} \tilde{\mathbf{u}}_{n}(t)\right) \mathrm{d} t$.

Lemma 6.

We have

$\liminf _{n \rightarrow \infty} \int_{0}^{T} j\left(\mathbf{u}_{n}(t), \frac{\mathrm{d}}{\mathrm{d} t} \tilde{\mathbf{u}}_{n}(t)\right) \mathrm{d} t \geq \int_{0}^{T} j(\mathbf{u}(t), \dot{\mathbf{u}}(t)) \mathrm{d} t$.

Proof.

We shall first show that

$$
\lim _{n \rightarrow \infty} \int_{0}^{T}\left[j\left(\mathbf{u}_{n}(t), \frac{\mathrm{d}}{\mathrm{d} t} \tilde{\mathbf{u}}_{n}(t)\right)-j\left(\mathbf{u}(t), \frac{\mathrm{d}}{\mathrm{d} t} \tilde{\mathbf{u}}_{n}(t)\right)\right] \mathrm{d} t=0 .
$$

By definition of $j$ we have

$$
\begin{aligned}
& \left|\int_{0}^{T}\left(j\left(\mathbf{u}_{n}(t), \frac{\mathrm{d}}{\mathrm{d} t} \tilde{\mathbf{u}}_{n}(t)\right)-j\left(\mathbf{u}(t), \frac{\mathrm{d}}{\mathrm{d} t} \tilde{\mathbf{u}}_{n}(t)\right)\right) \mathrm{d} t\right|=\left|\int_{0}^{T} \int_{Y_{3}}^{\mu}\left(\left|R \sigma_{\mathrm{N}}\left(\mathbf{u}_{n}(t)\right)\right|-\left|R \sigma_{\mathrm{N}}(\mathbf{u}(t))\right|\right)\left\|\frac{\mathrm{d}}{\mathrm{d} t} \tilde{\mathbf{u}}_{n_{\mathrm{T}}}(t)\right\|\right| \mathrm{d} t \mid \\
& \leq \int_{0}^{T} \int_{\Gamma_{3}} \mu\left|R \sigma_{\mathrm{N}}\left(\mathbf{u}_{n}(t)-\mathbf{u}(t)\right)\right|\left\|\frac{\mathrm{d}}{\mathrm{d} t} \tilde{\mathbf{u}}_{n_{\mathrm{T}}}(t)\right\| \mathrm{d} t \leq \int_{0}^{T}\left(\int_{\Gamma_{3}} \mu^{2}\left|R \sigma_{\mathrm{N}}\left(\mathbf{u}_{n}(t)-\mathbf{u}(t)\right)\right|^{2}\right)^{1 / 2}\left(\int_{\Gamma_{3}}\left\|\frac{\mathrm{d}}{\mathrm{d} t} \tilde{\mathbf{u}}_{n_{\mathrm{T}}}(t)\right\|^{2}\right)^{1 / 2} \mathrm{~d} t \\
& \leq \int_{0}^{T}|\mu(t)|_{L^{x}}\left\|R \sigma_{\mathrm{N}}\left(\mathbf{u}_{n}(t)-\mathbf{u}(t)\right)\right\|_{L^{2}\left(\Gamma_{3}\right)}\left\|\frac{\mathrm{d}}{\mathrm{d} t} \tilde{\mathbf{u}}_{n_{\mathrm{T}}}(t)\right\|_{L^{2}\left(\Gamma_{3}\right)} \mathrm{d} t \leq c \bar{\mu}\left\|R \sigma_{\mathrm{N}}\left(\mathbf{u}_{n}-\mathbf{u}\right)\right\|_{L^{2}(0, T)}\left\|\tilde{\mathbf{u}}_{n}\right\|_{W},
\end{aligned}
$$

where $\|\cdot\|_{L^{2}(0, T)}$ is the norm in $L^{2}\left(0, T ; L^{2}\left(\Gamma_{3}\right)\right)$. Thus we have

$$
\left.\left|\int_{0}^{T}\left(j\left(\mathbf{u}_{n}(t), \frac{\mathrm{d}}{\mathrm{d} t} \tilde{\mathbf{u}}_{n}(t)\right)-j\left(\mathbf{u}(t), \frac{d}{\mathrm{~d} t} \tilde{\mathbf{u}}_{n}(t)\right)\right) \mathrm{d} t\right| \leq \bar{c}\left\|R \sigma_{\mathrm{N}}\left(\mathbf{u}_{n}-\mathbf{u}\right)\right\|_{L^{2}(0, T}\right) .
$$


As $R$ is a compact operator $R \sigma_{\mathrm{N}}$ is therefore compact and as $\mathbf{u}_{n}(t)$ converges weakly towards $\mathbf{u}(t)$ for all $t \in[0, T], \lim _{n \rightarrow \infty}\left\|R \sigma_{\mathrm{N}}\left(\mathbf{u}_{n}-\mathbf{u}\right)\right\|_{L^{2}(0, T)}=0$.

We shall now show that

$$
\liminf _{n \rightarrow \infty} \int_{0}^{T} j\left(\mathbf{u}(t), \frac{\mathrm{d}}{\mathrm{d} t} \tilde{\mathbf{u}}_{n}(t)\right) \mathrm{d} t \geq \int_{0}^{T} j(\mathbf{u}(t), \dot{\mathbf{u}}(t)) \mathrm{d} t .
$$

Let $\boldsymbol{\Psi} \in L^{\infty}\left(0, T ;\left(L^{\infty}\left(\Gamma_{3}\right)\right)^{d}\right)$ be such that

$$
\boldsymbol{\Psi}(t)=\left\{\begin{array}{cl}
\frac{\dot{\mathbf{u}}_{\mathrm{T}}(t)}{\left|\dot{\mathbf{u}}_{\mathrm{T}}(t)\right|} & \text { if } \dot{\mathbf{u}}_{\mathrm{T}}(t) \neq \mathbf{0} \\
\mathbf{0} & \text { if not. }
\end{array}\right.
$$

Then $\mu\left|R \sigma_{\mathrm{N}}(\mathbf{u})\right| \boldsymbol{\Psi} \in L^{2}\left(0, T ;\left(L^{2}\left(\boldsymbol{\Gamma}_{3}\right)\right)^{d}\right)$ and using the Cauchy-Schwarz inequality in $\mathbf{R}^{d}$

$$
\begin{aligned}
\int_{0}^{T}\left(\mu\left|R \sigma_{\mathrm{N}}(\mathbf{u})\right| \boldsymbol{\Psi}(t), \frac{\mathrm{d}}{\mathrm{d} t} \tilde{\mathbf{u}}_{n_{\mathrm{T}}}(t)\right)_{\left(L^{2}\left(\mathbf{T}_{3}\right)\right)^{\prime}} \mathrm{d} t & =\int_{0}^{T} \int_{\Gamma_{3}}^{\mu}\left|R \sigma_{\mathrm{N}}(\mathbf{u})\right|\left(\boldsymbol{\Psi}(t), \frac{\mathrm{d}}{\mathrm{d} t} \tilde{\mathbf{u}}_{\mathrm{T}_{\mathrm{T}}}(t)\right)_{\mathbf{R}^{d}} \mathrm{~d} t \\
& \leq \int_{0}^{T} \int_{\Gamma_{3}} \mu\left|R \sigma_{\mathrm{N}}(\mathbf{u})\right|\left|\frac{\mathrm{d}}{\mathrm{d} t} \tilde{\mathbf{u}}_{n_{\mathrm{T}}}(t)\right| \mathrm{d} t,
\end{aligned}
$$

but

$$
\begin{aligned}
\lim _{n \rightarrow \infty} \int_{0}^{T}\left(\mu\left|R \sigma_{\mathrm{N}}(\mathbf{u})\right| \Psi(t), \frac{\mathrm{d}}{\mathrm{d} t} \tilde{\mathbf{u}}_{n_{\mathrm{T}}}(t)\right)_{\left(L^{2}\left(\Gamma_{3}\right)\right)^{i}} \mathrm{~d} t & =\int_{0}^{T}\left(\mu\left|R \sigma_{\mathrm{N}}(\mathbf{u})\right| \Psi(t), \dot{\mathbf{u}}_{\mathrm{T}}(t)\right)_{\left(L^{2}\left(\Gamma_{3}\right)\right)^{d i}} \mathrm{~d} t \\
& =\int_{0}^{T} \int_{\Gamma_{3}} \mu\left|R \sigma_{\mathrm{N}}(\mathbf{u})\right|\left(\boldsymbol{\Psi}(t), \dot{\mathbf{u}}_{\mathrm{T}}(t)\right)_{\mathbf{R}^{d}} \mathrm{~d} t \\
& =\int_{0}^{T} \int_{\Gamma_{3}} \mu\left|R \sigma_{\mathrm{N}}(\mathbf{u})\right|\left|\dot{\mathbf{u}}_{\mathrm{T}}(t)\right| \mathrm{d} t .
\end{aligned}
$$

So that we have

$$
\int_{0}^{T} \int_{\Gamma_{3}} \mu\left|R \sigma_{\mathrm{N}}(\mathbf{u})\right|\left|\dot{\mathbf{u}}_{\mathrm{T}}(t)\right| \mathrm{d} t \leq \liminf _{n \rightarrow \infty} \int_{0}^{T} \int_{\Gamma_{3}^{\prime}} \mu\left|R \sigma_{\mathrm{N}}(\mathbf{u})\right|\left|\frac{\mathrm{d}}{\mathrm{d} t} \tilde{\mathbf{u}}_{n_{\mathrm{T}}}(t)\right| \mathrm{d} t .
$$

By adding relations (4.6) and (4.7) proof of lemma 6 is achieved.

THEOREM 1.

$$
\begin{aligned}
& \text { The weak limit } \mathbf{u} \text { of } \mathbf{u}_{n} \text { satisfies the following inequality: } \forall \mathbf{v} \in L^{2}(0, T ; V) \\
& \begin{aligned}
\int_{0}^{T}(a(\mathbf{u}(t), \mathbf{v}(t)-\dot{\mathbf{u}}(t))+j(\mathbf{u}(t), \mathbf{v}(t))-j(\mathbf{u}(t), \dot{\mathbf{u}}(t))) \mathrm{d} t \\
\qquad \geq \int_{0}^{T}(\mathbf{f}(t), \mathbf{v}(t)-\dot{\mathbf{u}}(t)) \mathrm{d} t+\int_{0}^{T}\left\langle\sigma_{\mathrm{N}}(\mathbf{u}(t)), v_{\mathrm{N}}(t)-\dot{u}_{\mathrm{N}}(t)\right\rangle \mathrm{d} t
\end{aligned}
\end{aligned}
$$

and satisfies the unilateral condition

$\forall t \in[0, T] \quad \forall \mathbf{v} \in K \quad\left\langle\sigma_{\mathrm{N}}(\mathbf{u}(t)), v_{\mathrm{N}}-u_{\mathrm{N}}(t)\right\rangle \geq 0$.

Proof.

We begin by proving inequality (4.9). For this we shall return to the definition of the sequence $\mathbf{u}_{n}$ which is such that for all $t$ belonging to $\left.] 0, T\right], \mathbf{u}_{n}(t)$ satisfies the following inequality:

$$
\begin{aligned}
a\left(\mathbf{u}_{n}(t), \mathbf{w}-\mathbf{u}_{n}(t)\right)+j\left(\mathbf{u}_{n}(t), \mathbf{w}-\mathbf{u}_{n}\left(t-\frac{T}{n}\right)\right)-j\left(\mathbf{u}_{n}(t), \mathbf{u}_{n}(t)-\mathbf{u}_{n}\left(t-\frac{T}{n}\right)\right) & \\
& \geq\left(\mathbf{f}_{n}(t), \mathbf{w}-\mathbf{u}_{n}(t)\right) \quad \forall \mathbf{w} \in K,
\end{aligned}
$$


but for all $t$ belonging to $] 0, T]$ we have

$$
\left(\mathbf{u}_{n}\left(t-\frac{T}{n}\right)-\mathbf{u}(t), \boldsymbol{\varphi}\right) \leq\left\|\mathbf{u}_{n}\left(t-\frac{T}{n}\right)-\mathbf{u}_{n}(t)\right\|\|\boldsymbol{\varphi}\|+\left(\mathbf{u}_{n}(t)-\mathbf{u}(t), \boldsymbol{\varphi}\right) \quad \forall \varphi \in V
$$

and as by (4.4) we have

$$
\left\|\mathbf{u}_{n}\left(t-\frac{T}{n}\right)-\mathbf{u}_{n}(t)\right\| \leq C \int_{t-(T / n)}^{\min (t+(T / n) \cdot T)}\|\dot{\mathbf{f}}(\tau)\| \mathrm{d} \tau,
$$

we therefore obtain

$$
\left(\mathbf{u}_{n}\left(t-\frac{T}{n}\right)-\mathbf{u}(t), \boldsymbol{\varphi}\right) \leq\|\boldsymbol{\varphi}\| C \int_{t-(T / n)}^{\min (t+(T / n) \cdot T)}\|\dot{\mathbf{f}}(\tau)\| \mathrm{d} \tau+\left(\mathbf{u}_{n}(t)-\mathbf{u}(t), \boldsymbol{\varphi}\right) \quad \forall \boldsymbol{\varphi} \in V .
$$

So that $\mathbf{u}_{n}(t-(T / n))$ converges weakly to $\mathbf{u}(t)$ for all $\left.\left.t \in\right] 0, T\right]$ and by going to the limit in inequality (4.10) using relation (4.11), the properties of $a$ and $j$ and the weak convergence of $\mathbf{u}_{n}(t-(T / n))$ towards $\mathbf{u}(t)$ we obtain the following inequality for all $t \in[0, T]$ :

$$
a(\mathbf{u}(t), \mathbf{w}-\mathbf{u}(t))+j(\mathbf{u}(t), \mathbf{w}-\mathbf{u}(t)) \geq(\mathbf{f}(t), \mathbf{w}-\mathbf{u}(t)) \quad \forall \mathbf{w} \in K .
$$

Finally, by using Green's formula as in Section 2, one obtains inequality (4.9).

By going to the limit in inequality (4.5) using lemmas $4-6$ and the properties of continuity of $\sigma_{\mathrm{N}}$, one obtains the following inequality:

$$
\begin{aligned}
\int_{0}^{T}(a(\mathbf{u}(t), \mathbf{v}(t)-\dot{\mathbf{u}}(t))+j(\mathbf{u}(t), \mathbf{v}(t))-j(\mathbf{u}(t), \dot{\mathbf{u}}(t))) \mathrm{d} t \geq \int_{0}^{T}(\mathbf{f}(t), \mathbf{v}(t)-\dot{\mathbf{u}}(t)) \mathrm{d} t & \\
& \quad+\int_{0}^{T}\left\langle\sigma_{\mathrm{N}}(\mathbf{u}(t)), v_{\mathrm{N}}(t)\right\rangle \mathrm{d} t
\end{aligned}
$$

and then, as $\left\langle\sigma_{\mathrm{N}}(\mathbf{u}(t)), \dot{u}_{\mathrm{N}}(t)\right\rangle=0$ (see remark 2.1), one obtains inequality (4.8) which is a weak form of problem $P_{2}$. If we set in $(4.8) v \in L^{2}(0, T ; V)$ defined by:

$$
\mathbf{v}(s)= \begin{cases}\mathbf{w} & \text { for } s \in[t, t+h] \\ \mathbf{u}(s) & \text { otherwise }\end{cases}
$$

we obtain the following inequality:

$$
\begin{aligned}
\frac{1}{h} \int_{t}^{t+h}(a(\mathbf{u}(s), \mathbf{w}-\dot{\mathbf{u}}(s))+j(\mathbf{u}(s), \mathbf{w}) & -j(\mathbf{u}(s), \dot{\mathbf{u}}(s))) \mathrm{d} s \\
\geq & \frac{1}{h} \int_{t}^{t+h}(\mathbf{f}(s), \mathbf{w}-\dot{\mathbf{u}}(s)) \mathrm{d} s+\frac{1}{h} \int_{t}^{t+h}\left\langle\sigma_{\mathrm{N}}(\mathbf{u}(s)), w_{\mathrm{N}}-\dot{u}_{\mathrm{N}}(s)\right\rangle \mathrm{d} s
\end{aligned}
$$

and passing to the limit one obtains that $\mathbf{u}$ satisfies problem $P_{2}$ for almost all $t \in[0, T]$.

\section{ALGORITHM AND NUMERICAL APPLICATION}

\subsection{The discretized problem}

In order to solve the quasi variational inequality $S_{i}^{n}$ we introduce a fixed point method on the sliding limit, denoted by $g$ in the following: the quasi variational inequality is then replaced by a fixed point iteration on $g$, each iteration requires solving a variational inequality which can be considered as the variational form of a contact problem with Tresca friction. When the fixed point is reached the sliding limit shall be equal to $\mu\left|\sigma_{\mathrm{N}}(\mathbf{u})\right|$, which is the sliding limit given by the Coulomb friction law. Details can be found, for example, in Refs [16] and [17].

We use a finite element method ( $P 1$ elements) for solving each variational inequality which can be set as a minimization problem. Let $U^{h}$ (respectively $K^{h}$ ) be the finite dimensional space 
associated with discretization. The dimension of $U^{h}, M$, is equal if $d=2$ to twice the number of nodes.

At each fixed point iteration on $g$ we must solve the following discretized problems:

Problem $V_{i}^{n}$.

Find $\overline{\mathbf{u}}_{G}^{i+1} \in \prod_{j-1}^{M} K_{j}$ such that

$$
J_{1}\left(\overline{\mathbf{u}}_{G}^{i+1}\right) \leq J_{1}(\overline{\mathbf{v}}) \quad \forall \overline{\mathbf{v}} \in \prod_{j=1}^{M} K_{j}
$$

with

$$
J_{1}(\overline{\mathbf{v}})=\frac{1}{2} \overline{\mathbf{v}}^{\mathrm{T}} \mathbf{A} \overline{\mathbf{v}}-\left(\overline{\mathbf{F}}^{i+1}\right)^{\mathrm{T}} \overline{\mathbf{v}}+\left(\overline{\overline{\mathbf{G}}}^{i+1}\right)^{T}\left|\overline{\mathbf{v}}_{\mathrm{T}}-\overline{\mathbf{u}}_{\mathrm{T}}^{i}\right|,
$$

where $K_{j}=\mathbf{R}^{-}$if $j \in I_{\mathrm{c}}$ and $K_{j}=\mathbf{R}$ if $j \notin I_{\mathrm{c}}$ with $I_{\mathrm{c}}$ the set of integers corresponding to the degrees of freedom related to the normal component of the contact nodes and where the components of the vector $\overline{\overline{\mathbf{G}}}$ are given by $G_{\alpha}=\int_{\Gamma_{3}} g\left|\omega_{\alpha}\right|$ for $\alpha=1, \ldots$, card $I_{\mathrm{c}}$, where $\omega_{\alpha}$ is the shape function associated with the node $\alpha$.

Problem $V_{i}^{n}$ is a minimization problem under constraints of a quadratic functional including a non-differentiable term induced by the friction. The variables are the current displacements, the convex characterizing the constraints is independent of the evolution of the solution, the dependence on the loading history, i.e. the memory of the system induced by the friction, appears with the term $\overline{\mathbf{u}}_{\mathrm{T}}^{i}$ present in the expression (5.2) of $J_{1}(\overline{\mathbf{v}})$. These problems are solved through an iterative method such as the successive over-relaxation method with projection or a projected conjugate gradient method.

The fixed point problem is solved through a diagonal process which consists in doing rough resolutions for the first values of $g$ when solving problem $V_{i}^{n}$. The vector $\overline{\overline{\mathbf{G}}}^{i+1}$ is updated during the fixed point process by:

$$
\left(\overline{\overline{\mathbf{G}}}^{i+1}\right)^{m+1}=\mu\left|\sigma_{\mathrm{N}}\left(\overline{\mathbf{u}}_{G}^{i+1}\right)_{\mathrm{T}}^{m}\right|
$$

where the normal contact force is computed directly from the values of the defect of equilibrium.

\section{REMARK 5.1.}

The alternative incremental formulation given in Refs [16] and [18] can be deduced from problem $S_{i}^{n}$ by keeping the increments $\Delta \overline{\mathbf{u}}_{G}^{i+1}$ as variables (setting $\overline{\mathbf{u}}_{G}^{i+1}=\overline{\mathbf{u}}_{G}^{i}+\Delta \overline{\mathbf{u}}_{G}^{i+1}$ ). In that case problem $V_{i}^{n}$ is written as follows.

PRoblem $\boldsymbol{W}_{i}^{n}$.

Find $\Delta \overline{\mathbf{u}}_{G}^{i+1} \in \prod_{j=1}^{M} K_{j}^{i}$ such that

with

$$
J_{2}\left(\Delta \overline{\mathbf{u}}_{G}^{i+1}\right) \leq J_{2}(\overline{\mathbf{v}}) \quad \forall \overline{\mathbf{v}} \in \prod_{j=1}^{M} K_{j}^{i}
$$

$$
J_{2}(\overline{\mathbf{v}})=\frac{1}{2} \overline{\mathbf{v}}^{\mathrm{T}} \mathbf{A} \overline{\mathbf{v}}-\left(\overline{\mathbf{R}}^{i}+\Delta \overline{\mathbf{F}}^{i+1}\right)^{\mathrm{T}} \overline{\mathbf{v}}+\left(\overline{\overline{\mathbf{G}}}^{i+1}\right)^{\mathrm{T}}\left|\overline{\mathbf{v}}_{\mathrm{T}}\right|,
$$

where the convex depends on the solution obtained at the previous step and is given by

$$
K_{j}^{i}=\left\{\begin{array}{c}
\mathbf{R} \text { if } j \notin I_{\mathrm{c}} \\
\left\{\alpha ;\left(\alpha+v_{j}^{i}\right) \leq 0\right\} \text { if } j \in I_{\mathrm{c}}
\end{array}\right.
$$

$\overline{\mathbf{R}}^{i}=\overline{\mathbf{F}}^{i}-\mathbf{A} \overline{\mathbf{u}}^{i}$ being the previous defect of equilibrium (equal to the contact forces).

In this case the variables are the displacement increments, the convex depends at each step on the solution $\overline{\mathbf{u}}^{i}$ and the dependence on the loading history appears with the extra term $\overline{\mathbf{R}}^{i}$ in the loading which represents the contact forces at the previous time step $t_{i}$. 


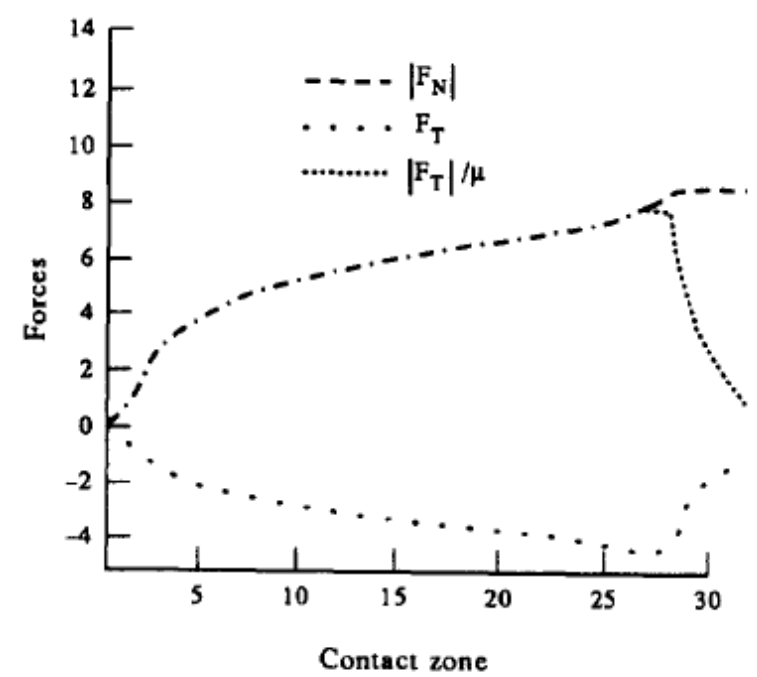

Fig. 1.

\subsection{Numerical example}

In this paper a very simple example is given to illustrate the dependence of the solution on the loading history. However, a few industrial applications can be found in the following papers: assembly of a pressure vessel with a sequential loading in Ref. [18], as well as various examples such as the punch problem with loading and unloading, structure assembly and a cracked viscoplastic solid under alternative loading in Ref. [16].

The following example is a simple benchmark example that was proposed by a group in charge of validating computer codes in the GRECO "Grandes déformations et Endommagement".

A long bar is squeezed onto a plane rigid obstacle by applying a vertical force $f$ and a symmetrical horizontal force $F$ at each side of the bar. We give in Figs 1 and 2 the contact forces on one side of the contact edge (because of the symmetry) obtained for Fig. 1 by applying the vertical force $f$ first and then applying $F$, and for Fig. 2 we start by applying the horizontal force $F$ before applying $f$. The sliding part of the contact edge is characterized by the superposition of the plots of $F_{\mathrm{N}}$ and $F_{\mathrm{T}} / \mu$ (Coulomb criterion). Only two time steps were carried out. The mesh contained 230 nodes, of which 32 were contact nodes. Figure 3 shows that loading by sequence 1 gives, as one may expect, a very different solution from the one obtained by the sequence 2 loading, although the loading at the final time step is the same. The

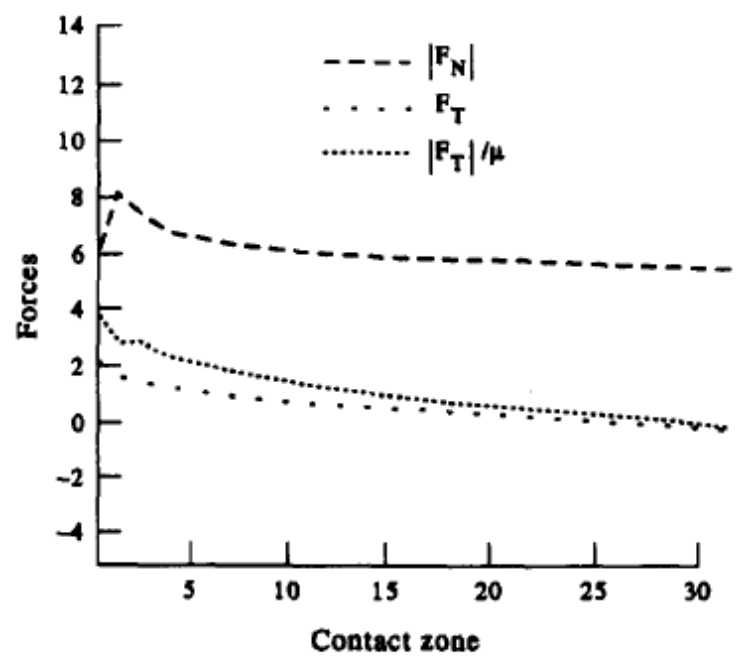

Fig. 2. 


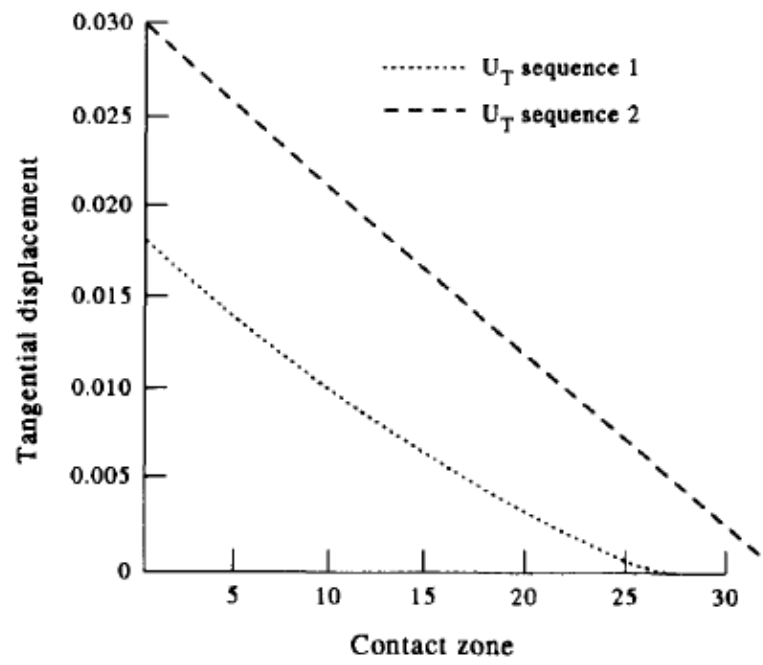

Fig. 3.

state of the contact forces at the final step shows that part of the contact zone is sliding for sequence 1 (the Coulomb criterion is met on the left part of the zone) whereas all the contact zone is blocked for sequence 2 (the criterion is never met).

\section{REFERENCES}

[1] M. COCU, E. PRATT and M. RAOUS, C.R. Acad. Sci. Paris Série I 320, 1413 (1995).

[2] M. COCU, E. PRATT and M. RAOUS, in Proc. Contact Mechanics International Symposium-Carry-Le-Rouet, p. 13. Plenum, New York (1995).

[3] J. T. ODEN and J. A. C. MARTINS, Comput. Meth. Appl. Mech. Engng 52, 527 (1985).

[4] N. KIKUCHI and J. T. ODEN, Contact Problems in Elasticity SIAM, Philadelphia (1988).

[5] A. KLARBRING, A. MIKELIC and M. SHILLOR, Int. J. Engng Sci. 26, 811 (1988).

6] A. KLARBRING, A. MIKELIC and M. SHILLOR, Nonlinear Analysis, Theory Meth. Applic. 13, 935 (1989).

7] J. A. C. MARTINS and J. T. ODEN, Nonlinear Analysis, Theory Meth. Applic. 11, 407 (1987).

[8] L. E. ANDERSSON, Nonlinear Analysis, Theory Meth. Applic. 16, 347 (1991).

[9] A. KLARBRING, A. MIKELIC and M. SHILLOR, in International Series of Numerical Mathematics, Vol. 101. p. 85. Birkhäuser, Basel (1991).

[10] G. DUVAUT, C.R. Acad. Sci. Paris Série A 290, 263 (1980).

[11] L. DEMKOWICZ and J. T. ODEN, Nonlinear Analysis, Theory Meth. Applic. 6, 1075 (1992).

[12] J. T. ODEN and E. PIRES, J. Appl. Mech. 50, 67 (1983).

[13] T. CAZENAVE and A. HARAUX, Introduction aux Problèmes d'Evolution Semilinéaires. Ellipses, Paris (1990).

[14] G. DUVAUT and J.-L. LIONS, Les Inéquations en Mécanique et en Physique. Dunod, Paris (1972).

[15] M. COCU, Int. J. Engng Sci. 22, 567 (1984).

[16] M. RAOUS, P. CHABRAND and F. LEBON, J. Mécanique Théorique Appliquée, supl. 1 to Vol. 7, 111 (1988).

[17] C. LICHT, E. PRATT and M. RAOUS. in International Series of Numerical Mathematics, Vol. 101, p. 129. Birkhäuser, Basel (1991).

[18] F. LEBON and M. RAOUS, Comput. Struct. 43, 925 (1992). 